University of New Hampshire

University of New Hampshire Scholars' Repository

Space Science Center

Institute for the Study of Earth, Oceans, and

Space (EOS)

7-29-2010

\title{
Development of the Advance Energetic Pair Telescope (AdEPT) for medium-energy gamma-ray astronomy
}

\author{
Stanley D. Hunter \\ NASA Goddard Space Flight Ctr \\ Peter F. Bloser \\ University of New Hampshire, Peter.Bloser@unh.edu \\ Michael P. Dion \\ NASA Goddard Space Flight Ctr \\ Mark L. McConnell \\ University of New Hampshire - Main Campus, mark.mcconnell@unh.edu \\ Georgia A. DeNolfo \\ NASA Goddard Space Flight Ctr
}

See next page for additional authors

Follow this and additional works at: https://scholars.unh.edu/ssc

Part of the Astrophysics and Astronomy Commons

\section{Recommended Citation \\ Stanley D. Hunter ; Peter F. Bloser ; Michael P. Dion ; Mark L. McConnell ; Georgia A. de Nolfo ; Seunghee Son ; James M. Ryan and Floyd W. Stecker "Development of the Advance Energetic Pair Telescope (AdEPT) for medium-energy gamma-ray astronomy", Proc. SPIE 7732, Space Telescopes and Instrumentation 2010: Ultraviolet to Gamma Ray, 773221 (July 29, 2010); doi:10.1117/12.857298; http://dx.doi.org/10.1117/12.857298 \\ This Conference Proceeding is brought to you for free and open access by the Institute for the Study of Earth, Oceans, and Space (EOS) at University of New Hampshire Scholars' Repository. It has been accepted for inclusion in Space Science Center by an authorized administrator of University of New Hampshire Scholars' Repository. For more information, please contact Scholarly.Communication@unh.edu.}




\section{Authors}

Stanley D. Hunter, Peter F. Bloser, Michael P. Dion, Mark L. McConnell, Georgia A. DeNolfo, Seunghee Son, James M. Ryan, and Floyd Stecker 


\title{
Development of the Advanced Energetic Pair Telescope (AdEPT) for Medium-Energy Gamma-Ray Astronomy
}

\author{
Stanley D. Hunter*a, Peter F. Bloser ${ }^{\mathrm{b}}$, Michael P. Dion ${ }^{\mathrm{a}}$, Mark L. McConnell ${ }^{\mathrm{b}}$, \\ Georgia A. de Nolfo ${ }^{a}$, Seunghee Son ${ }^{\mathrm{a}}$, James M. Ryan ${ }^{\mathrm{b}}$, and Floyd W. Stecker ${ }^{\mathrm{a}}$ \\ a NASA/Goddard Space Flight Center, Code 661, Greenbelt, MD 20771 \\ b Department of Physics, University of New Hampshire, Durham, NH 03824
}

\begin{abstract}
Progress in high-energy gamma-ray science has been dramatic since the launch of INTEGRAL, AGILE and FERMI. These instruments, however, are not optimized for observations in the medium-energy $\left(\sim 0.3<\mathrm{E}_{\gamma}<\sim 200 \mathrm{MeV}\right)$ regime where many astrophysical objects exhibit unique, transitory behavior, such as spectral breaks, bursts, and flares. We outline some of the major science goals of a medium-energy mission. These science goals are best achieved with a combination of two telescopes, a Compton telescope and a pair telescope, optimized to provide significant improvements in angular resolution and sensitivity. In this paper we describe the design of the Advanced Energetic Pair Telescope (AdEPT) based on the Three-Dimensional Track Imager (3-DTI) detector. This technology achieves excellent, mediumenergy sensitivity, angular resolution near the kinematic limit, and gamma-ray polarization sensitivity, by high resolution 3 -D electron tracking. We describe the performance of a $30 \times 30 \times 30 \mathrm{~cm}^{3}$ prototype of the AdEPT instrument.
\end{abstract}

Keywords: Gamma-ray, telescope, imaging, medium-energy, pair production, time projection chamber, micro-well readout

\section{INTRODUCTION}

Since the launch of INTEGRAL, AGILE, and FERMI, progress in low-energy (35 keV $\left.<\mathrm{E}_{\gamma}<\sim 10 \mathrm{MeV}\right)$ and highenergy $\left(\sim 20 \mathrm{MeV}<\mathrm{E}_{\gamma}>10 \mathrm{GeV}\right)$ gamma-ray astronomy has been, and will continue to be dramatic. The instruments, which make up these missions, cover broad energy ranges, however, none is optimized for observations in the mediumenergy $\left(\sim 0.3<\mathbf{E}_{\gamma}<\sim 200 \mathrm{MeV}\right)$ regime where many astrophysical objects exhibit unique transitory behavior and spectral features, e.g. breaks, bursts and flares, and nuclear lines. While continued progress from current observations is expected, there will remain a significant sensitivity gap in medium-energy observations. The last several NASA decadal surveys have supported the idea of a medium-energy mission because of the richness of this spectral domain.

We envision this medium-energy gamma-ray mission to consist of two co-aligned, wide field-of-view telescopes, a Compton scattering telescope and a pair production telescope, optimized for excellent angular resolution with a significant improvement in sensitivity. Achieving significant improvements in sensitivity in the medium-energy regime requires low background and large effective area as well as high angular resolution and energy resolution especially for point or line sources.

In this paper we briefly highlight some aspects of medium-energy gamma-ray science and describe the development of the Advance Energetic Pair Telescope (AdEPT), a pair production telescope that provides the significant improvements needed for a medium-energy gamma-ray mission.

\footnotetext{
*stanley.d.hunter@nasa.gov; phone 301-286-7280; fax 301-286-1682
}

Space Telescopes and Instrumentation 2010: Ultraviolet to Gamma Ray, 


\section{MEDIUM-ENERGY GAMMA-RAY SCIENCE}

Much of the matter observed in the Universe today has been through a spectacular lifecycle. Since formation of stars and galaxies shortly after the Big Bang, this matter has undergone stellar formation and evolution ending in novae or supernovae that eject energetic particles and nuclei forming new generations of matter and stars. The goal of mediumenergy gamma-ray astrophysics is a direct insight into this cycle by measuring the gamma-ray emissions.

At $\mathrm{MeV}$ energies, the Universe is nearly transparent. A future instrument with increased sensitivity can provide a view of the most distant objects in the Universe. The key for a future mission will be to provide adequate sensitivity and the best angular resolution to go beyond simply detecting sources to making detailed spatially, spectrally and temporally resolved measurements of the high-energy Universe.

Medium-energy gamma-ray astronomy has the potential to make important contributions to several long-standing problems central to modern physics, astrophysics, and cosmology. Potential contributions include the detection of dark matter annihilation processes [1] [2], the measurement of the extragalactic background radiation fields and the strength of cosmic magnetic fields, detailed information about the astrophysical processes that lead to the growth of black holes, information about the earliest epoch of star-formation [3], and the study of the most extreme particle accelerators in the Universe. Exploring the 5-30 MeV energy range opens up a variety of new phenomena for study, e.g. micro-quasars, colliding wind binary systems, etc. We highlight a few of these subjects that will be addressed by significant improvements in angular resolution.

Galactic Diffuse Emission: The positrons responsible for the $511 \mathrm{keV}$ gamma-ray emission from the Galactic center [4] can have many origins. One of these is likely to be energetic point sources, e.g. pulsars. Accurate maps of candidate positron sources in the Galaxy are necessary to understand this emission.

Photons from interstellar space in the 5 to $\sim 100 \mathrm{MeV}$ energy range reflect the transition from electron dominated to hadron dominated processes. The one spectral feature in the high-energy gamma-ray spectrum, the pion-bump, was predicted by Morrison [5] and Stecker [6], and confirmed by EGRET [7]. Mapping this emission on an angular scale better than $0.5^{\circ}$ at $100 \mathrm{MeV}$ will allow individual supernova remnants (SNRs), the boundaries of supper bubbles, and large-scale Galactic structure to be delineated and examined for evidence of cosmic ray acceleration and propagation in our Galaxy.

Pulsars: The energy range from 0.1 to $100 \mathrm{MeV}$ is a critical one for pulsar studies. Theoretical models predict a transition at $\sim 10 \mathrm{MeV}$ between two components of the pulsar spectrum, viz., a higher energy one produced by primary electrons and a lower energy one produced by cascade pair electrons and positrons [8]. These two components are predicted to have different spectral and polarization characteristics. No X-ray or gamma-ray pulsar polarization measurements presently exist, even for the Crab pulsar.

Supernova Remnants: The turbulent nature of SNRs [9] suggests that small-scale structures, i.e. knots, are sites for particle acceleration. New breakthroughs in Imaging Air Cherenkov Telescope (IACT) have registered photon signals from many potential sites of cosmic-ray acceleration, with spatially and spectrally resolved $\mathrm{TeV}$ images of shell-type SNR and pulsar wind nebulae [10]. However, even with the excellent spectral and spatial resolution of these measurements, there is still no smoking-gun signature for hadronic processes ( $\pi^{0}$-emission) to distinguish it from leptonic (inverse Compton) emission. Differentiating between these two processes can be accomplished with a gammaray instrument with angular resolution several times better than FERMI for energies below $\sim 200 \mathrm{MeV}$ where the pion signature resides.

Extragalactic Diffuse Gamma-Ray Emission: The extragalactic diffuse gamma-ray spectrum below $30 \mathrm{MeV}$ is relatively poorly measured and not well understood [11][12]. Observations in this range are important for searching for signals from, or providing constraints on, the existence of new particles, such as the gravitino and graviton, which have been predicted by some extra-dimension models. The objects and physical processes that produce the extragalactic diffuse gamma-ray emission below $30 \mathrm{MeV}$ may be different from those that produce the lower energy X-ray background spectrum and the higher energy background gamma-ray spectrum. There has been much progress in understanding the origin of the diffuse extragalactic gamma-ray spectrum in the range below $200 \mathrm{keV}$, now believed to be from radio-quiet active galactic nuclei (AGN) or Seyfert galaxies, and, in the range above $30 \mathrm{MeV}$, is now believed to be from beamed radio-loud AGN, i.e. blazars. However, the gap below $\sim 30 \mathrm{MeV}$ may provide a window into other astrophysical processes. Improved angular resolution over the energy gap will help resolve sources that can be removed from the true extragalactic emission. 
Active Galactic Nuclei \& Gamma-Ray Blazars: There is little information about AGNs below $100 \mathrm{MeV}$. COMPTEL measurements of $3 \mathrm{C} 273$ indicate that some AGN can be most luminous from 10 to $30 \mathrm{MeV}$ rather than the well studied range above $100 \mathrm{MeV}$. Their frequency, spatial distribution, and variability are poorly known, much less understood. Expanding our coverage of the AGN population in this little explored region will provide new insights into the demographics and nature of these objects. The gamma-ray emission mechanism for blazars whose energy spectra peak in the $\mathrm{MeV}$ range may involve synchrotron radiation in which case a second peak from Compton radiation may exist in the very high energy $\mathrm{TeV}$ range. A promising test for synchrotron emission from blazars, e.g. 3C273 [13], comes from measuring the gamma-ray polarization.

Testing Relativity with Polarization: Galactic pulsars such as the Crab Nebula pulsar are known to exhibit strong polarization from synchrotron emission. In all gamma-ray sources, the observation of polarization enables a better understanding of the nature of the gamma-ray emission. However, in the case of extragalactic sources such as GRBs and blazars, detection of polarization has a broader fundamental physics implication. In the general formulation of effective field theory for QED with Planck-scale Lorentz invariance violation, a vacuum birefringence effect, akin to Faraday rotation, is present causing photons of opposite helicity to have different phase velocities, an effect which increases with energy [14]. The difference in the photon rotation angles grows linearly with time and therefore with the distance to the source. This will cause any intrinsic source polarization to be washed out by the time the gamma-ray photons reach Earth from large distances. Thus, the perfect candidate sources for testing this violation of relativity are sources at cosmological distances such as GRBs and blazars. If gamma-ray polarization is detected in such sources, this limits the Lorentz invariance violation. Such limits will have implications for quantum gravity theories and string theories. Testing Lorentz invariance is not only a fundamental question in physics, but it has also gained attention recently in the theoretical physics community because of its importance in developing a quantum theory of gravity and the nature of space-time at the Planck scale [15].

Solar Flares: Above $10 \mathrm{MeV}$ solar flare emissions come from primary electron Bremsstrahlung and a mixture of secondary radiation of nuclear origin. Identifying and quantifying the separate components places strong constraints on acceleration and transport processes at the Sun. A major high-energy solar-flare question is the nature of the acceleration process during so-called long duration solar gamma-ray flares [16][17]. With $100 \mathrm{MeV}$ emission persisting for hours in several historic and large flare events, the acceleration process must take place over large volumes over an extended time. Ryan \& Lee [18] modeled these events with second order Fermi acceleration in large coronal loops. Recently, however, Kahler \& Ragot [19] have rejuvenated an interplanetary shock model where protons accelerated by the shock diffuse back to the Sun. The loop model should produce wide but distinct, and perhaps moving, emission regions, while diffuse emission is expected over much of the solar disk if particles are diffusing backwards from a shock, probably larger than one solar radius in size. Thus, imaging the solar disk at $\sim 100 \mathrm{MeV}$ would be crucial to understanding this emission and would constitute a breakthrough observation.

\section{MEDIUM-ENERGY TELESCOPE CONCEPT}

Detection methods in the medium-energy range are as diverse as the physical processes that produce those gamma rays. The medium-energy regime includes photons that interact with matter predominantly via photo-electric absorption, Compton scattering, and pair production. Sensitive detection (direction, energy, and polarization) of photons over this three-plus decade range in energy cannot be accomplished with a single instrument. At the low end of the scale, the photoelectric effect has been used effectively in space at $500 \mathrm{keV}$ and below for transient measurements and for steadystate sources with collimated/shielded spectrometers. Above this energy, up to $\sim 30 \mathrm{MeV}$, Compton scatter telescopes have best preferred since pair telescopes, e.g. EGRET, have not been able operate below $\sim 30 \mathrm{MeV}$. Based on crosssections however, Compton telescopes are best suited only up to $\sim 10 \mathrm{MeV}$ while pair production telescopes are best above $\sim 5 \mathrm{MeV}$. We have demonstrated the viability of pair production imaging at $6.13 \mathrm{MeV}$, far lower than the EGRET threshold, with a 3-DTI prototype. For an intermediate energy mission, AdEPT offers significant energy overlap with a Compton telescope, may take advantage of both pair and Compton processes, as well as providing exceptional angular resolution above $\sim 20 \mathrm{MeV}$ via pair production. A Compton telescope compatible with this mission concept is described by Bloser et al. [20].

The medium-energy sky is rich in objects and structure. Advancing our scientific understanding requires gamma-ray telescopes with optimum angular resolution and improved sensitivity. Sensitivity is determined by the effective area and angular resolution, which are not independent. Increasing the effective area of a telescope improves sensitivity by collecting more photons. The angular resolution must also improve; otherwise source confusion will limit the scientific return.

Proc. of SPIE Vol. 7732 773221-3 

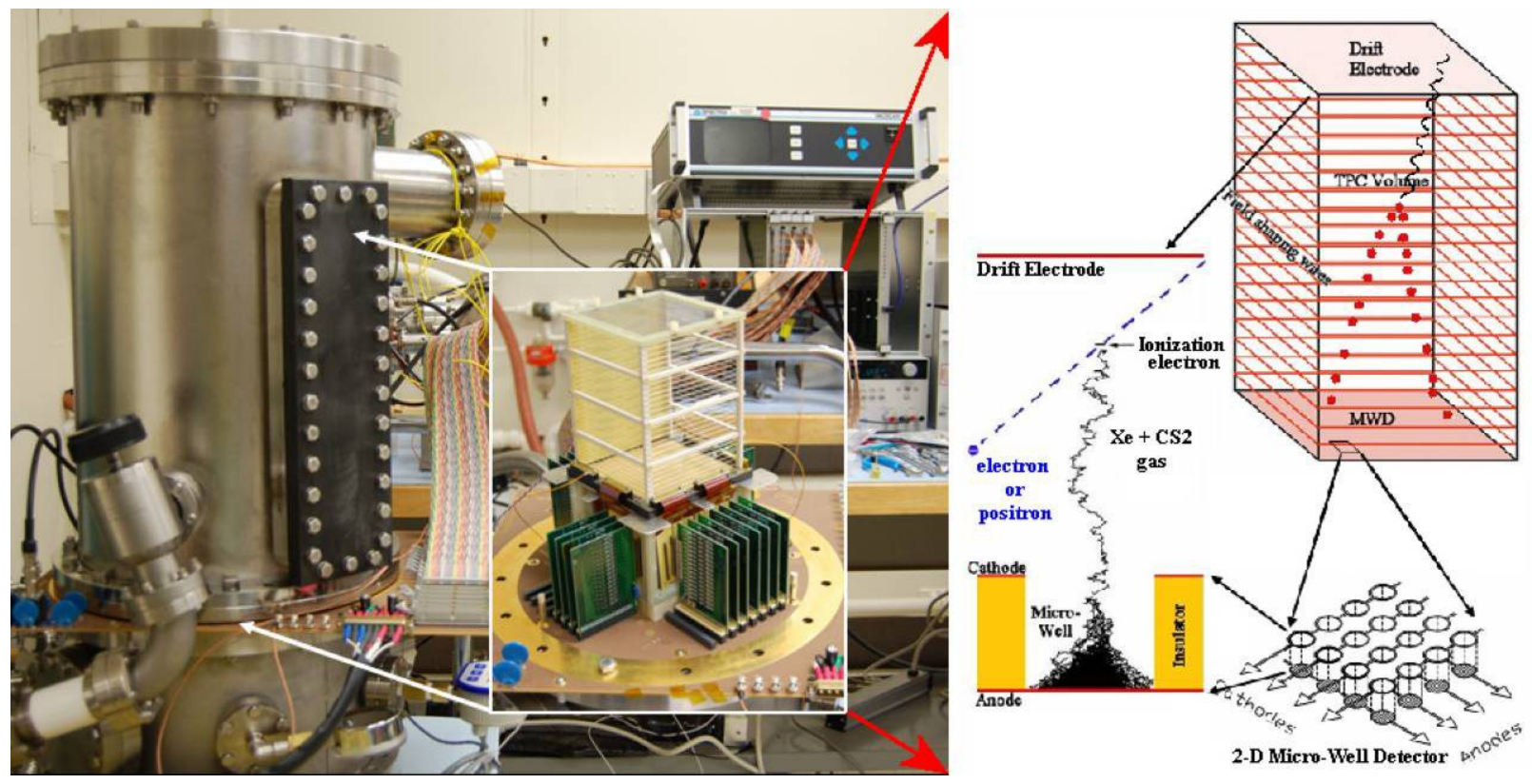

Figure $1-10 \times 10 \times 15 \mathrm{~cm}^{3} 3$-DTI prototype (inset photo) and vacuum chamber (left). Schematic diagram of the 3DTI TPC, MWD and simulation of an electron induced avalanche in a micro-well (right).

The telescopes required to address the medium-energy science described above can be expressed in terms of the instrument performance: 1) Large field of view, $2 \pi \mathrm{sr}$ (hemispherical), 2) Uniform sensitivity (homogenous detector), 3) Able to handle background from cosmic ray interactions in the instrument and spacecraft, and 4) High angular resolution and polarization sensitivity. The 3-DTI detector described here meets the above performance requirements.

\subsection{3-DTI Technology}

Three-Dimensional Track Imager (3-DTI) technology, Figure 1, is being developed for medium- and high-energy gamma-ray telescopes with excellent gamma-ray angular resolution [21][22][23][24]. Because photons can only be detected via their charged interaction byproducts with matter, this requires a detector with high granularity, i.e. highresolution three-dimensional electron tracking in a low density medium. The 3-DTI combines a time projection chamber (TPC), consisting of a gas volume bounded by a drift electrode at the top and field shaping wires on the sides, with a two-dimensional micro-well detector (MWD) on the bottom. The $0.4 \mathrm{~mm}$ spatial resolution of the MWD combined with the homogenous gaseous medium provides the high granularity required for tracking the electron-positron pair from gamma-ray pair interactions. Electron tracking also enables measurement of gamma-ray polarization, a new tool to study astrophysical phenomenon [25].

The MWD consists of orthogonal anode and cathode electrodes on the bottom and top of a polyimide sheet, respectively. The micro-wells, essentially blind vias laser micro-machined concentric with holes in the cathode electrodes, through the polyimide, exposing a portion of the anode electrodes at the bottom of the well. A uniform electric field, $\sim 1 \mathrm{kV} / \mathrm{cm}$, between the drift grid and the cathode electrodes provides the drift field whereas a much stronger field, $\sim 20 \mathrm{kV} / \mathrm{cm}$, in the micro-wells provides proportional gas gain of $\sim 10^{4}$. The charge collected on each anode and the image charge on each cathode are continuously converted to a voltage signal and digitized at $2.5 \times 10^{6} \mathrm{samples} / \mathrm{s}$.

The time correlation of the anode and cathode charge pulses determines the X-and Y-coordinate of the charge and the arrival time provides the Z-coordinate. Collection of all the charge along a track provides a 3-D image of the particle track. Separate digitization of all anode and cathode signals insures that multiple charged particles traversing the TPC volume can be tracked simultaneously. The pulse height is proportional to the $\mathrm{dE} / \mathrm{dx}$ energy loss of the particle.

The raw data for one et-pair recorded from a $6.13 \mathrm{MeV}$ gamma ray $\left({ }^{238} \mathrm{Pu} /{ }^{13} \mathrm{C}\right.$ source) interacting in $80 \% \mathrm{P}-10+20 \%$ $\mathrm{CS}_{2}, 0.55 \mathrm{~atm}$, is shown in Figure 2. The drift field was $880 \mathrm{~V} / \mathrm{cm}$ and MWD field was $4 \times 10^{4} \mathrm{~V} / \mathrm{cm}$. The structure visible in the raw TD data, corresponding to the MWD avalanche signals of ionization electron clouds, illustrates that the 3-DTI 
is capable of recording the track with high resolution including the statistics of the ionization process, energy loss mechanism, and Molière scattering, Figure 4. Additional events, Figure 3, were definitely electron tracks associated with

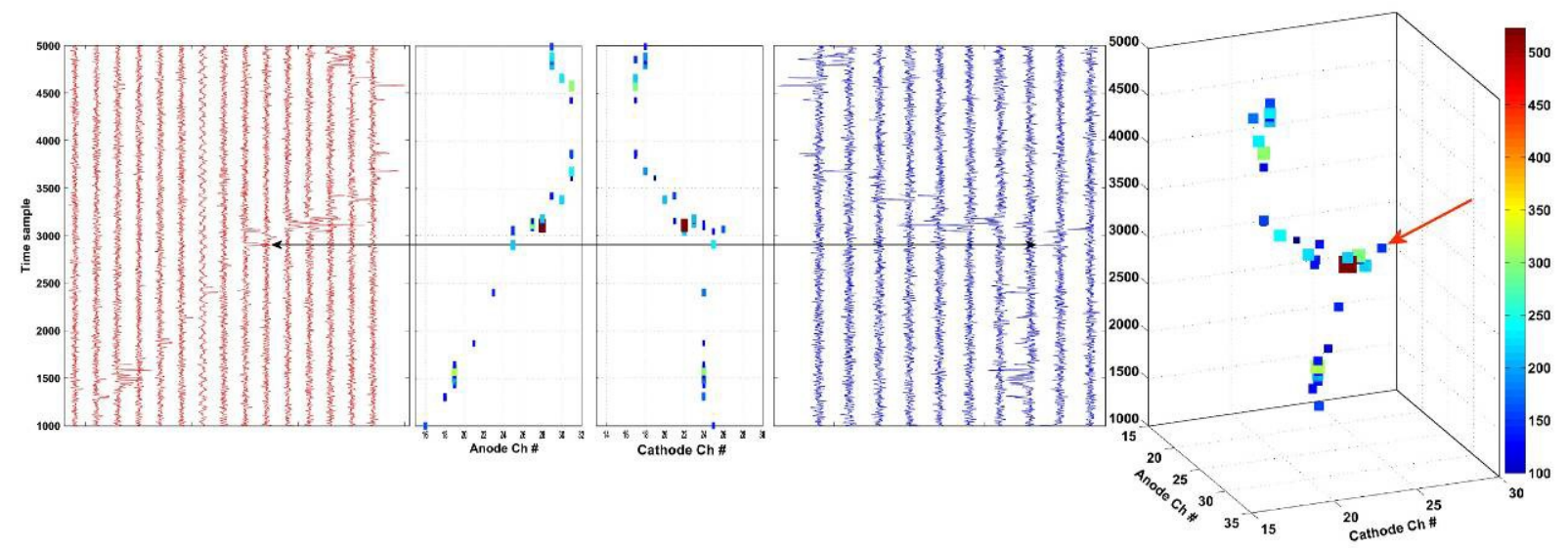

Figure 2 - The raw 3-DTI data from the anodes (red) and cathodes (blue) and 2-D voxelized data for a $6.13 \mathrm{MeV}$ gamma ray. The red arrow in the 3-D projection indicates our best estimate of the incident gamma ray direction.

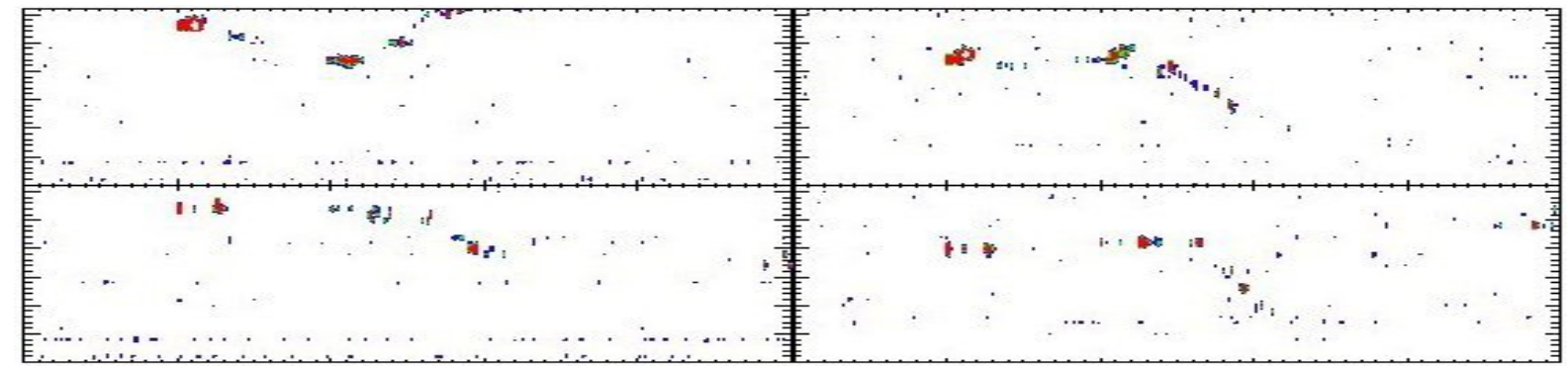

Figure 3 - Additional $6.13 \mathrm{MeV}$ gamma ray events. Anode, $x$-z projection (left), cathodes, $y$-z projection (right).

the gamma-ray source, however, understanding the energy deposition, and identifying the vertex of the interaction suffers from marginal gain of the MWD. Despite these uncertainties, we have reconstructed the thirteen best vertex candidates to get an indication of incident gammaray direction from the source placed $10 \mathrm{~cm}$ above the active volume. This preliminary result, imaging of gamma rays in an energy range that overlaps with Compton scattering telescopes promises, is very promising for a future medium-energy mission.

Negative ion drift is the key to the large 3-DTI drift length with minimal diffusion and low power [26][27][28]. The negative ion drift velocity is $2-4$ orders of magnitude slower than electron drift and the diffusion is reduced nearly to the low thermal limit, Figure 5. We use carbon disulfide, $\mathrm{CS}_{2}$, as the negative ion component in 3-DTI. The slow drift velocity reduces the sample rate of the transient digitizer, which, in turn, reduces the power per channel of the readout electronics, and thermal diffusion allows for much longer drift distances.

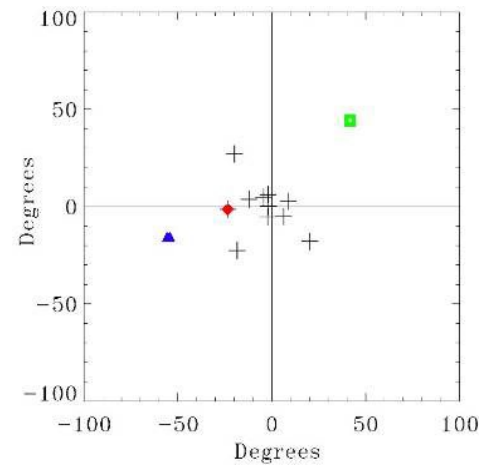

Figure 4 - Incident $6.13 \mathrm{MeV}$ gamma-rays reconstructed for thirteen best vertex events. The colored symbols are for the events shown in Figures 2 and 3. No correction has been made for parallax. 

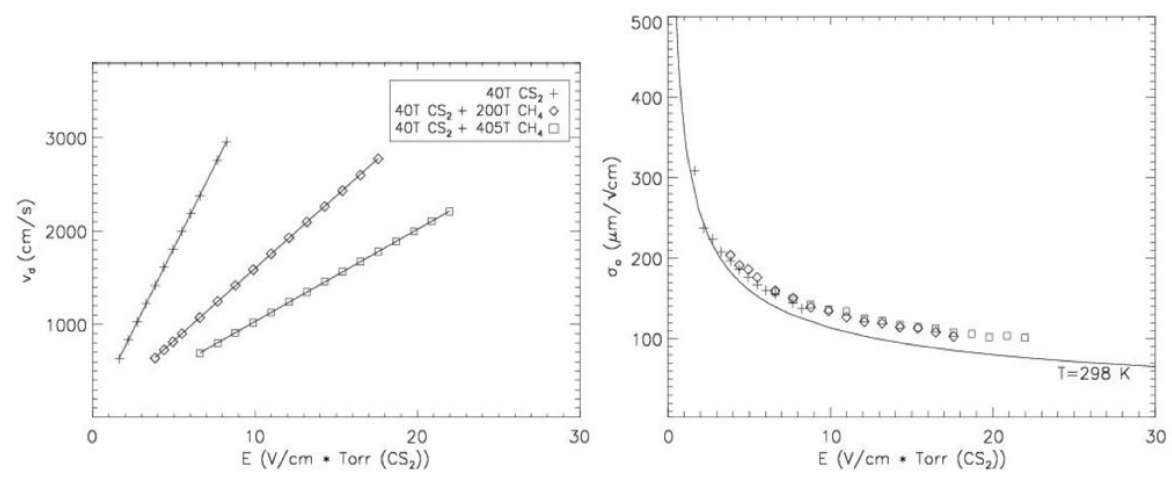

Figure 5 - The negative ion drift velocity in $\mathrm{CS}_{2}$ at 40 torr and $\mathrm{CH}_{4}$ at 0,200 , and 400 torr as a function of the reduced field (left). The solid lines are linear fits to the data points. The longitudinal diffusion for the same gas mixtures as a function of reduced field (right). The solid line is the thermal diffusion expected at $298 \mathrm{~K}$. The symbols used in the two plots correspond to the same $\mathrm{CH}_{4}$ pressures.
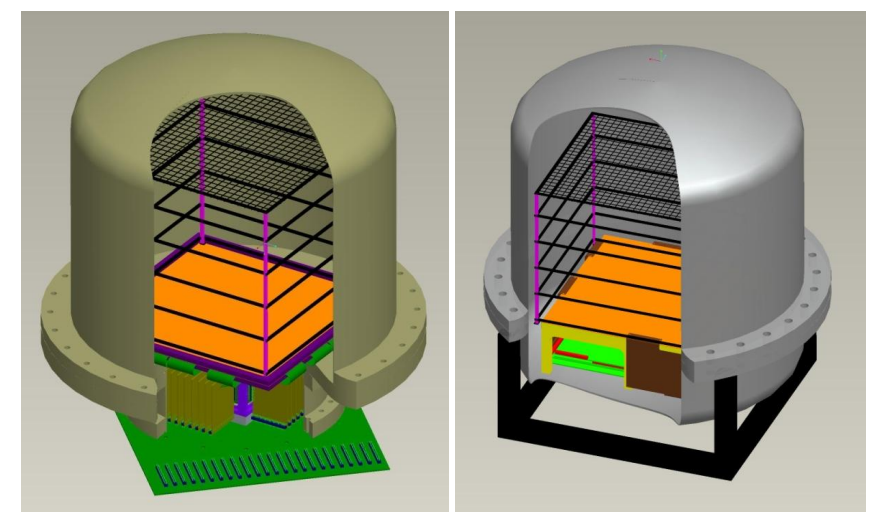

Figure 6 - The $30 \mathrm{~cm}$ prototype will be developed in three steps. Step one (left) uses discrete front-end and digitizer electronics. Steps two and three (right) introduce second generation digitizer and ASIC front-end electronics respectively.
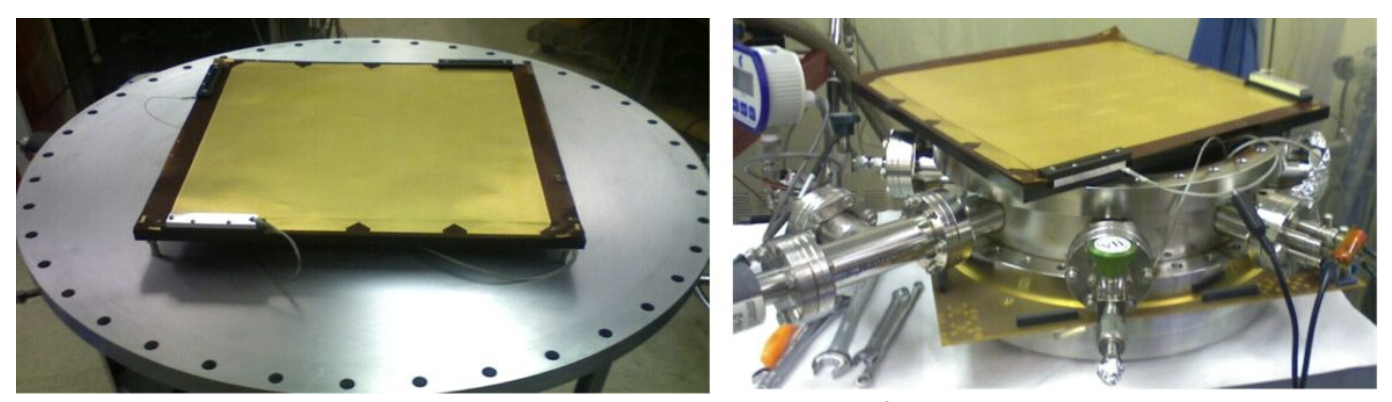

Figure 7 - Assembly of the $30 \mathrm{~cm}$ prototype hardware. The $30 \times 30 \mathrm{~cm}^{2} \mathrm{MWD}$ is visible on top of parts of the gas pressure chamber. 


\section{$3.230 \mathrm{~cm}$ Prototype}

The $30 \times 30 \times 30 \mathrm{~cm}^{3}$ prototype, hereafter referred to as the $30 \mathrm{~cm}$ prototype, is shown schematically in Figure 6 , and during construction in Figure 7. The $30 \mathrm{~cm}$ prototype is being developed in three steps. Step one uses the discrete frontend and digitizer electronics of the $10 \mathrm{~cm}$ prototype. Step two adds a second generation digitizer that will allow continuous readout and eliminate nearly all instrumental dead-time. The third step will replace the discrete front-end electronics with an ASIC. We are completing assembling of the step one prototype and will start lab testing in the June timeframe using atmospheric muons, electrons, and $6.13 \mathrm{MeV}$ gammas. The third step will be self-contained in a robust pressure vessel that will be suitable for testing at accelerators.

\section{CONCLUSION}

We have demonstrated gamma-ray imaging at $6.13 \mathrm{MeV}$ in $\mathrm{CH}_{4}$ with the 3-DTI detector and have spent a year optimizing the fabrication of high-gain MWDs and scaling up the 3-DTI hardware to $30 \mathrm{~cm}$. We are now poised to acquire more extensive gamma ray data this summer with the $30 \mathrm{~cm}$ prototype. A sub-orbital proposal is planned for the 2013 time-frame with a $50 \mathrm{~cm}$ AdEPT prototype.

\section{REFERENCES}

[1] Rudaz, S., \& Stecker, F. W. ApJ, 325, 16 (1988)

[2] Rudaz, S., \& Stecker, F. W. ApJ, 368, 406 (1991)

[3] Stecker, F. W. (2007) "Stellar Photon and Blazar Archaeology with Gamma-rays"

[4] Purcell, W. R., et al. ApJ, 491, 725 (1997)

[5] Morrison, P., Novio Cimento, 7, 858 (1958)

[6] Stecker, F. W. ApJ, 212, 60 (1977)

[7] Hunter, S.D., et al. ApJ, 481, 205 (1997)

[8] Harding, A. K., et al. ApJ, 680, 1378 (2008)

[9] Jokipii, J. R., \& Giacalone, J. ApJ, 660, 336 (2007)

[10] Aharonian, F., et al. A\&A, 457, 899 (2006)

[11] Sreekumar, P., Stecker, F. W., \& Kappadath, S. C. "Proceedings of the Fourth Compton Symposium, Williamsburg, VA" AIP Conf. Proc. 410, 344 (1997)

[12] Stecker, F. W., \& Salamon, M. H. [The Extragalactic Gamma-Ray Background], 432 (2001)

[13] Collmar, W., et al. "The Fifth Compton Symposium, Portsmouth, NH” AIP, 510, 591 (2000)

[14] Jacobson, T., Liberati, S., Mattingly, D., \& Stecker, F. W. Phys. Rev. Let., 93, 2, (2004)

[15] Mattingly, D. "Living Reviews in Relativity" 8, 5 (2005)

[16] Ryan, J. M., et al. Bulletin of the American Astronomical Society, 32, 847, (2000)

[17] Chupp, E. L., \& Ryan, J. M., Research in Astron. Astrophys. 9/1, 11. (2009)

[18] Ryan, J. M., \& Lee, M. A. ApJ, 368, 316, (1991)

[19] Kahler, S. W., \& Ragot, B. R. ApJ, 675, 846 (2008)

[20] Bloser, P.F., et al. these proceedings (2010)

[21] Hunter, S.D., et al. IEEE NSS, San Diego, CA, 778 - 782, 2006,

[22] Son, S. et al., IEEE NSS, N14-113 (2006)

[23] de Nolfo, G.A., et al. Proc. SPIE, Vol. 6954, 695404 (2008)

[24] Hunter, S.D., et al. Proc. SPIE, Vol. 6954, 695415 (2008)

[25] Bloser, P. F., Hunter, S. D., Depaola, G. O. \& Longo, F. SPIE, X-Ray and Gamma-Ray Instrumentation for Astronomy XIII, 5165, 322-333 (2004)

[26] Martoff, C. J., et. al. Nucl. Instr. and Meth. A, 440(2):355-359, (2000)

[27] Miyamoto, J., et. al. Nucl. Instr. and Meth. A, 526:409-412, (2004)

[28] Martoff, C. J., et. al. Nucl. Instr. and Meth. A, 555:55-58, (2005) 